\title{
Photodynamic Therapy for the Treatment of Precancerous Lesions
}

\author{
B.N.Radhika ${ }^{1}$, Dr. Arathy S. Lankupalli \\ (C.R.R.I, Department of Oral Medicine and Radiology, Saveeetha University, India) \\ (M.D.S, Senior Lecturer, Department of Oral Medicine and Radiology, Saveeetha University, India)
}

\begin{abstract}
Photodynamic therapy (PDT), also known as photoradiation therapy, phototherapy or photochemotherapy ${ }^{I}$ is a minimally invasive surgical technique, whose application in dentistry is growing rapidly to target the premalignant and malignant disorders in the head and neck, gastrointestinal tract, lungs and skin with reduced levels of morbidity. It involves the use of a photoactive dye (photosensitizer) that is activated by exposure to light of a specific wavelength in the presence of oxygen. The transfer of this energy from the activated photosensitizer to available oxygen results in the formation of toxic oxygen ions like free radicals and singlet oxygen ${ }^{3}$. These released reactive chemical ions can damage proteins, lipids and nucleic acids ${ }^{3}$. This review article lays emphasis on the basic aspects of photodynamic therapy and the various researches done regarding the treatment of premalignant lesions using this new therapeutic approach.
\end{abstract}

Keywords: Photodynamic therapy, Photosensitizers, Premalignant lesions.

\section{Introduction}

Potentially malignant disorders, previously known as potentially malignant lesions or precancerous lesions are identified by exclusion of other white and red lesions ${ }^{2}$. It refers to precancer as not all disorders described under this term may transform into cancer. Leukoplakias and erytrhoplakias represent the majority of these disorders ${ }^{2}$. The others include lichen planus, oral submucous fibrosis, xeroderma pigmentosum, actinic cheilitis etc ${ }^{2}$.

The management of these precancerous lesions is not well defined ${ }^{2}$. It largely depends upon the risk or exposure factors, complete removal of the lesion followed by continuous monitoring after the removal ${ }^{1,2}$. The first choice of treatment for these disorders that have progressed to malignancy has always been a surgical approach, but now PDT has been recognized as an alternative treatment modality for their management.

\subsection{A brief note on photodynamic therapy}

Photodynamic therapy (PDT) is an emerging treatment modality that employs the photochemical interaction of three components: light, photosensitizer, and oxygen ${ }^{1}$. Tremendous progress has been made in the last 2 decades in new technical development of all components as well as understanding of the biophysical mechanism of PDT.

It is a minimally invasive therapeutic modality approved for clinical treatment of several types of cancer and non-oncological disorders. The subsequent activation of the PS by visible light preferentially in the red region of the visible spectrum where the tissues are more permeable to light generates reactive oxygen species, responsible for cytotoxicity of neoplastic cells and tumor regression ${ }^{1,4}$. There are three main mechanisms described by which oxygen contributes to the destruction of tumors by PDT: direct cellular damage, vascular shutdown and activation of immune response against tumor cells. It can be targeted accurately sparing the normal tissues, can be done in an out-patient lab, day care setting and is convenient for the patient ${ }^{4}$. The main advantages in using the PDT are high efficacies for small superficial tumors, little or no scarring and the repetition of treatment without cumulative toxicity ${ }^{4}$. These aspects favor PDT, as an alternate treatment modality to conventional approach in the management of oral pre malignant and malignant lesions.

\section{Discussion}

\subsection{Principle mechanism of PDT}

As already discussed above, the three fundamentals of PDT are oxygen, a photo sensitizer and visible light. Activation of photo sensitizer upon absorption of light results in excitation of the drugs from its ground state into a singlet state (reactive singlet oxygen). This moiety is highly cytotoxic, with a short life time and a short radius of action ${ }^{5}$. The direct cytotoxic activity and microvascular damage contribute to the destruction of tumor cells. This tissue eventually sloughs away or is resorbed and there is normal healing and reepithelialization of the treated site. 
There are two mechanisms by which the triplet state photo sensitizer can react with bio-molecule.

Type - I: Involves electronic hydrogen transfer directly from the photo sensitizer, producing ions which remove the hydrogen /electron from the substrate molecule to form a free molecule. These radicals react rapidly with oxygen, resulting in the production of highly reactive oxygen species (hydrogen peroxide, superoxide, hydroxyl radicals) ${ }^{1,4}$.

Type - II: These produce electronically excited and highly reactive state of oxygen known as singlet oxygen. This is the most damaging species generated during $\mathrm{PDT}^{1,4}$.

Both the mechanisms of damage are dependent on both oxygen tension and photo sensitizer concentration. The photo sensitizer can be administered systemically or topically. After injection, a photosensitizer is initially detained within the tumor vasculature, and PDT which utilizes a short drug to light interval damages mainly the tumor vasculature and a long drug to light interval allowed for the diffusion of the photo sensitizer into the tissue, facilitates its accumulation into cellular compartments, and more direct tumor cytotoxicity ${ }^{1}$. PDT produces cytotoxic effect through photodamage to subcellular organelles and molecules. Mitochondria, lysosomes, cell membranes, and nuclei of tumor cells are considered potential targets, along with tumor vasculature. During light exposure, sensitizers that localize in mitochondria may induce apoptosis, while sensitizers that localize in lysosomes and cell membranes may induce necrosis ${ }^{1}$. After illumination of the photo sensitizer, an acute stress response leads to changes in calcium and lipid metabolism, and production of cytokines and stress proteins ${ }^{1}$. PDT generates measureable changes in tumor oxygen and blood flow during illumination. Damage to the tumor vascular network can diminish the supply of oxygen to the tumor. In addition, the production of reactive oxygen species is associated with utilization of oxygen. This process, known as photochemical oxygen consumption, can also generate hypoxia during treatment ${ }^{1}$. This illumination induced hypoxia can further reduce the tumor response. Because PDT is a cold photochemical process, there is no tissue heating, and connective tissues such as collagen and elastin are largely unaffected ${ }^{2}$.

\subsection{Photo Sensitizers}

Photosensitizers are probably incorporated directly into the cellular membrane, but they do not seem to accumulate within the cell nuclei. They are taken up and retained preferentially by neo plastic tissue, but as yet this effect is not sufficiently pronounced to allow a selective clinical response. The only three photosensitizers that have received approval by regulatory authorities are Photofrin (PorfimerSodium), 5- Aminolaevulanic Acid (ALA) and Verteporfin (BPD, Benzzoporphyrinderivative).

These various agents can be grouped under three generations:

2.2.1 First generation: Photofrin - most extensively used in the treatment of early cancers of stomach, lungs and head and neck.

a) Hematoporphyrin derivatives (HPD)

2.2.2 Second Generation: Aminolaevulanic Acid (ALA) - a naturally occurring precursor in the heme biosynthetic pathway, has received approval only for a non-malignant disorder, actinic keratosis.

a) Benzoporphyrin derivative(BPD)

b) M-Tetrohydroxyphenylchlorin ( $\mathrm{mTHPC})$ and

c) Talaporphin sodium (LS11)

2.2.3 Third Generation: Include currently available drugs that are modified by targeting with monoclonal antibodies or with non-antibody-based protein carriers and protein/ receptor systems, and conjugation with a radioactive $\operatorname{tag}^{4,6,7}$.

\subsection{PDT: Light Sources}

PDT requires a source of light that activates the photosensitizer by exposure to low power visible light at a specific wavelength ${ }^{4}$. Human tissue transmits red light efficiently, and the longer activation wavelength of the photosensitizer results in deeper light penetration ${ }^{4}$. Different techniques are used to illuminate the tumor. These include superficial, interstitial, intra-operative and intra-cavitary PDT. Photosensitizer activation was achieved via a variety of light sources, like argon pumped dye lasers, potassium titanylphosphate (KTP) or neodymium:yttrium aluminium garnet( $\mathrm{Nd} / \mathrm{YAG})$-pumped dye lasers, and gold vapor or copper vapor-pumped dye lasers. The main disadvantages with these systems were that they were expensive and complex to handle ${ }^{4}$.The presently used systems are easy to handle, portable, and cost-effective. Non-coherent light sources like tungsten-filament, quartz halogen, xenon arc, metal halide, and phosphor-coated sodium lamps, are in use. Non laser light sources, such as light-emitting diodes (LED), have also been applied in PDT ${ }^{4}$. 


\subsection{Role of PDT In Oral Pre-Malignancies}

2.4.1 Oral leukoplakia:The term leukoplakia should be used to recognize white plaques of questionable risk having excluded known diseases or disorders that carry no increased risk for cancer ${ }^{8}$. This clinical entity may reflect a spectrum of histologic diagnosis ranging from para-keratosis to dysplasia and microinvasive carcinoma ${ }^{8}$. The application of photodynamic therapy in oral leukoplakia significantly reduces the time of treatment in comparison with pharmacological method involving Vitamin- $\mathrm{A}^{3}$.

The PDT agent most commonly used is 5-ALA at concentration of 10-20\%. $0.1 \%$ chlorophyll gel is being used too $^{3}$. Both oral and topical ALA is used for oral dysplatic lesions. 5-ALA is converted in-situ to a photosensitizer, protoporphyrin-IX, in the heme biosynthetic pathway. The light penetration is limited at $635 \mathrm{~nm}$ which restricts the use of ALA to superficial lesions ${ }^{3,4}$.

2.4.2 Oral erythroplakia, Verrucous hyperplasia: Oral erythroplakia has been considered the oral mucosal lesion with the greatest potential for malignant transformation in the mouth. It is defined as a 'fiery red patch' that cannot be characterized clinically or pathologically as any other definable disease ${ }^{8}$.Topical ALAPDT using the $635 \mathrm{~nm}$ light emitting diode(LED) light is very effective for the treatment of oral erythroplakia and verruous hyperplasia. The verrucous appearance of the $\mathrm{OVH}$ lesion provides a large area for retention and absorption of ALA on the surface ${ }^{3,4}$.

2.4.3 Lichen planus: It is a relatively common chronic inflammatory mucocutaneous disease demonstrating immune pathology $y^{8}$. T-cell mediated autoimmune phenomena are involved in the pathogenesis of lichen planus $^{3}$.The mechanism of PDT for the treatment of lichen planus remains unclear but it appears to act on hyperproliferating cells, such as those present in malignancies which selectively uptake the photosensitizers ${ }^{3}$. It may have an immunomodulatory effects and may induce apoptosis in the hyperproliferating inflammatory cells, which are present in psoriasis and lichen planus. This may reverse the hyperproliferation and inflammation of lichen planus ${ }^{3}$.The agent used could be methylene blue(MB-PDT).

\section{Conclusion}

PDT has achieved significant clinical benefits and improvement in the quality of life in patients who suffer from premalignanies and advanced oral malignancies. The advantages compared to surgery or radiotherapy are reduced long term morbidity, non-invasive technique, convenient technique for the patient, economical, excellent cosmetic results etc. The recent developments in photosensitizers and light delivery systems have substantially reduced treatment times and reduced photosensitivity, while increasing the achievable depth of necrosis. The main drawback against using PDT as frontline therapy lies in the fact that large randomized trials have not yet been done. Treatment regimens still have to be optimized and standardized for better therapeutic effectiveness ${ }^{3}$. Although PDT has shown its potential in the management of early stage oral malignancies with a very good outcome it is still considered as an adjunct to surgical intervention. It is still not considered as a single stand-alone treatment modality for various disorders ${ }^{2}$. In summary, premalignant changes in the mouth which are often widespread are frequently lasered or excised. PDT is only another option in the treatment regimen, on its own or in combination with other modalities and after further researches and studies could become the main treatment option for various premalignant disorders.

\section{References}

[1] Photodynamic Therapy in Dentistry, K. Konopka and T. Goslinski, J Dent Res 86(8):694-707, 2007

[2] Photodynamic Therapy in the management of Potentially Malignant and Malignant OralDisorders.WaseemJerjes, Zaid Hamdoon and Colin Hopper. Jerjes et al. Head \& neck oncology 2012, 4:16. http://www.headandneckoncology.org/content/4/1/16

[3] Photodynamic therapy in Oral Diseases. Sudhakara Reddy .R ,Ramya . Kotha , Ramesh Tatapudi , SubbarayuduGudapati , Sai Madhavai .N, Sai Kiran .CH. Int J Biol Med Res. 2012; 3(2): 1875-1883.

[4] Photodynamic therapy- A Novel Therapeutic approach in the management of Oral Premalignant and Malignant lesions. Mubeen, Kavitha. M. AOSR 2011;1(4):228-234

[5] Overholt bf, Panjehpour M. Photodynamic Therapy for barrett's esophagus: Follow-up in 100 patients. gastrointestendosc1999; 49:1-7.

[6] Kriegmair M, Baumgartner R, Lumper w, et al. Early clinical experience with 5-aminolevulinic acid for the photodynamic therapy of superficial bladder cancer. br j urol1996; 77: 667-71.

[7] Fijan S, Honigsmann H, Ortel B. Photodynamic therapy of epithelial skin tumours using delta-aminolaevulinic acid and desferrioxamine. br j dermatol1995; 133: 282-88.

[8] Nomenclature and classification of potentially malignant disorders of the oral mucosa. S.warnakulasuriya, Newell. W. Johnson, I.van derwaal. j oral pathol med(2007) 36:575-80. 